MATHEMATICS OF COMPUTATION

Volume 72, Number 244, Pages 1841-1854

S 0025-5718(03)01542-4

Article electronically published on May 20, 2003

\title{
A PSEUDOSPECTRAL MAPPING THEOREM
}

\author{
S.-H. LUI
}

\begin{abstract}
The pseudospectrum has become an important quantity for analyzing stability of nonnormal systems. In this paper, we prove a mapping theorem for pseudospectra, extending an earlier result of Trefethen. Our result consists of two relations that are sharp and contains the spectral mapping theorem as a special case. Necessary and sufficient conditions for these relations to collapse to an equality are demonstrated. The theory is valid for bounded linear operators on Banach spaces. For normal matrices, a special version of the pseudospectral mapping theorem is also shown to be sharp. Some numerical examples illustrate the theory.
\end{abstract}

\section{INTRODUCTION}

The spectrum of a normal matrix paints an accurate picture of the behavior of the matrix. Here, normality refers to the matrix having a complete set of orthogonal eigenvectors. The spectrum of a nonnormal matrix, however, may not be very informative. Thanks largely to the work of Trefethen and his coworkers ([2], 4]), the pseudospectrum has emerged as an appropriate indicator for the stability of nonnormal systems. It has been applied to problems in hydrodynamic instability, turbulence, magnetohydrodynamics, control theory, the iterative solution of linear equations, the numerical solution of differential equations, quantum mechanics, random matrices, etc. See the review articles mentioned above for references.

For a matrix $A$ and nonnegative number $\epsilon$, recall that the $\epsilon$-pseudospectrum of $A$ can be defined as the following closed set in the complex plane:

$$
\Lambda_{\epsilon}(A) \equiv \bigcup_{\|E\| \leq \epsilon} \Lambda(A+E) .
$$

Here $\Lambda()$ denotes the spectrum of a matrix and $\|\cdot\|$ is the matrix 2-norm. When $A$ is a normal matrix, its $\epsilon$-pseudospectrum is the union of closed disks of radius $\epsilon$ with centers at the eigenvalues. For a nonnormal matrix, its $\epsilon$-pseudospectrum can be much bigger than this union.

The spectral mapping theorem is a fundamental result in functional analysis of great importance. Given a matrix $A$ and a function $f$ which is analytic on an open set containing $\Lambda(A)$, the theorem asserts that

$$
f(\Lambda(A))=\Lambda(f(A)) .
$$

Received by the editor October 11, 2001 and, in revised form, March 29, 2002.

2000 Mathematics Subject Classification. Primary 15A18, 15A60, 65F15.

Key words and phrases. Pseudospectra, eigenvalues, spectral mapping theorem.

This work was supported in part by a grant from NSERC.

(C)2003 American Mathematical Society 
In [1], the authors consider sixteen pseudospectral theorems that generalize corresponding spectral theorems: each $\epsilon$-pseudospectral theorem reduces to an eigenvalue theorem when $\epsilon=0$. Theorem $14 \epsilon$ in this collection states that $\Lambda_{\epsilon|\beta|}(\alpha+$ $\beta A)=\alpha+\beta \Lambda_{\epsilon}(A)$ for $\alpha, \beta$ complex numbers. When $\epsilon=0$, this is the spectral mapping theorem for affine functions. Actually, this result had already appeared earlier

in $[3$. The goal of this paper is to extend this mapping theorem for pseudospectra to analytic functions.

The following is an outline of the paper. In Section 2, the general pseudospectral mapping theorem in the form of two set inclusions is stated and proved. It is shown that the set inclusions reduce to an equality for all matrices iff the mapping is an affine function. A weaker version of the pseudospectral mapping theorem that turns out to have interesting consequences is demonstrated in Section 3. In Section 4, an adaptation of this "weak" theorem for normal matrices is shown to be sharp. This is followed by some numerical experiments which illustrate the theory in the final section.

In the remainder of this introduction, we offer two simple examples to illustrate the role of and motivation for studying pseudospectra. First consider a system of ordinary differential equations $x^{\prime}(t)=f(x(t))$. Suppose $f(0)=0$ and $D f(0)$, the matrix of partial derivatives of $f$, has eigenvalues whose real parts are all negative. Then it is well understood that 0 is an (asymptotically) stable equilibrium point in the sense of Liapunov. If $D f(0)$ is a highly nonnormal matrix, then the pseudospectrum of $D f(0)$ may protrude far into the right half of the complex plane (assuming that $D f(0)$ has one eigenvalue not too far away from the imaginary axis). While eigenvalue analysis predicts stability, pseudospectral analysis predicts instability which is usually the more acceptable answer unless we are only interested in the ideal system without noise or other errors.

Next, consider the system $x^{\prime}(t)=f(x(t), \lambda)$, where $\lambda$ is a real parameter. Suppose for all $\lambda$ in some neighborhood containing $0, f(0, \lambda)=0$ and $D f(0, \lambda)$ has eigenvalues $\alpha(\lambda) \pm i \beta(\lambda)$, where $\alpha$ and $\beta$ are real. Suppose further that at $\lambda=0$ there is exactly one pair of pure imaginary eigenvalues $\pm i \beta(0)$, where $\beta(0)$ is positive, and $d \alpha / d \lambda \neq 0$. Then the celebrated Poincare-Andronov-Hopf bifurcation theorem states that there exists a branch of time-periodic solutions in a neighborhood of $(x, \lambda)=(0,0)$ of period approximately $2 \pi / \beta(0)$. However, if $D f(0,0)$ is highly nonnormal, then a slight perturbation of $D f(0,0)$ may lead to no periodic solution or to a periodic solution with a period possibly very different from $2 \pi / \beta(0)$.

\section{Pseudospectral MAPPing THEOREM}

The following is a pseudospectral mapping theorem for complex analytic functions. It is sharp in the sense that the pair of functions $(\phi, \psi)$ defining the sizes of the pseudospectra are optimal. Actually, the theorem is an easy consequence of the definitions of these functions.

Theorem 2.1. Let $A$ be a matrix and let $f$ be an analytic function defined on $\mathcal{D}$, an open set containing $\Lambda(A)$. For each $\epsilon, s \geq 0$ and sufficiently small, define

$$
\phi(\epsilon)=\sup _{\zeta \in \Lambda_{\epsilon}(A)} \inf \left\{r \geq 0, f(\zeta) \in \Lambda_{r}(f(A))\right\}
$$

and

$$
\psi(s)=\sup _{z \in \Lambda_{s}(f(A))} \inf \left\{r \geq 0, z \in f\left(\Lambda_{r}(A)\right)\right\} .
$$


Then

$$
f\left(\Lambda_{\epsilon}(A)\right) \subset \Lambda_{\phi(\epsilon)}(f(A)) \subset f\left(\Lambda_{\psi(\phi(\epsilon))}(A)\right) .
$$

Proof. We first show that $\phi$ is well defined. Let $\zeta \in \Lambda_{\epsilon}(A)$. Then $\zeta \in \Lambda(A+E)$ for some matrix $E$ such that $\|E\| \leq \epsilon$. By the spectral mapping theorem,

$$
f(\zeta) \in \Lambda(f(A+E))=\Lambda(f(A)+F),
$$

where $F=f(A+E)-f(A)$ and satisfies $\|F\| \leq r$ for some positive $r$. We may take $r$ to be independent of $\zeta$ since $\zeta \in \Lambda_{\epsilon}(A)$. Thus $f(\zeta) \in \Lambda_{r}(f(A))$ which implies that the infimum in the definition of $\phi$ is taken over a nonempty set and thus $\phi$ is well defined and $\phi(\epsilon) \leq r$. The first set inclusion now follows directly from the definition of $\phi$.

Next we show that $\psi$ is well defined. Let $z \in \Lambda_{s}(f(A))$. Then $z \in \Lambda(f(A)+F)$ for some matrix $F$ such that $\|F\| \leq s$. Let $z=f(\zeta)$ for some $\zeta \in \mathcal{D}$. (If such $\zeta$ does not exist, then $z \notin f(\mathcal{D})$ which is impossible if $s$ is small enough.) Therefore $z \in f\left(\Lambda_{r}(A)\right)$ for some positive $r$ which may be taken to be independent of $z \in$ $\Lambda_{s}(f(A))$. Thus the infimum in the definition of $\psi$ is taken over a nonempty set and so $\psi$ is well defined and $\psi(s) \leq r$. The second set inclusion now follows directly from the definition of $\psi$ with $s=\phi(\epsilon)$. This completes the proof.

Here are some remarks.

(1) Note that $\phi(0)=0=\psi(0)$ and thus the theorem reduces to the usual spectral mapping theorem when $\epsilon=0$.

(2) If $f(z)=\alpha+\beta z$, where $\alpha, \beta$ are complex numbers, then

$$
\begin{aligned}
\phi(\epsilon)= & \sup _{\zeta \in \Lambda_{\epsilon}(A)} \inf \{r \geq 0, \alpha+\beta \zeta \in \Lambda(\alpha+\beta A+F), \\
& \text { some } F \text { such that }\|F\| \leq r\} \\
= & \sup _{\zeta \in \Lambda_{\epsilon}(A)} \inf \{r \geq 0, \alpha+\beta \zeta \in \alpha+\beta \Lambda(A+F / \beta), \\
= & \text { some } F \text { such that }\|F\| \leq r\} \\
= & \sup _{\zeta \in \Lambda_{\epsilon}(A)} \inf \left\{r \geq 0, \alpha+\beta \zeta \in \alpha+\beta \Lambda_{r /|\beta|}(A)\right\} \\
=\epsilon|\beta| . &
\end{aligned}
$$

In a similar way, $\psi(s)=s /|\beta|$ and hence $\psi(\phi(\epsilon))=\epsilon$, recovering Theorem $14 \epsilon$ in 1. Here, we have assumed $\beta \neq 0$; otherwise, it is trivially true.

(3) By the definitions of $\phi$ and $\psi$, the set inclusions are sharp in the sense that the functions cannot be replaced by smaller functions.

(4) It is well known that an equivalent definition of the $\epsilon$-pseudospectrum is

$$
\Lambda_{\epsilon}(A)=\left\{z \in \mathbb{C},\left\|(z-A)^{-1}\right\| \geq \epsilon^{-1}\right\},
$$

where the norm is taken to be $\infty$ if $z \in \Lambda(A)$. It follows that

$$
\phi(\epsilon)=\sup _{\zeta \in \Lambda_{\epsilon}(A)}\left\|(f(\zeta)-f(A))^{-1}\right\|^{-1}
$$

and

$$
\psi(s)=\sup _{z \in \Lambda_{s}(f(A))} \inf _{\zeta \in f^{-1}(z)}\left\|(\zeta-A)^{-1}\right\|^{-1} .
$$


The following is a short justification.

$$
\begin{aligned}
\phi(\epsilon) & =\sup _{\zeta \in \Lambda_{\epsilon}(A)} \inf \left\{r \geq 0, f(\zeta) \in \Lambda_{r}(f(A))\right\} \\
& =\sup _{\zeta \in \Lambda_{\epsilon}(A)} \inf \left\{r \geq 0,\left\|(f(\zeta)-f(A))^{-1}\right\| \geq r^{-1}\right\} \\
& =\sup _{\zeta \in \Lambda_{\epsilon}(A)}\left\|(f(\zeta)-f(A))^{-1}\right\|^{-1} .
\end{aligned}
$$

Similarly,

$$
\begin{aligned}
\psi(s) & =\sup _{z \in \Lambda_{s}(f(A))} \inf \left\{r \geq 0, z \in f\left(\Lambda_{r}(A)\right)\right\} \\
& =\sup _{z \in \Lambda_{s}(f(A))} \inf \left\{r \geq 0, \zeta \in f^{-1}(z),\left\|(\zeta-A)^{-1}\right\| \geq r^{-1}\right\} \\
& =\sup _{z \in \Lambda_{s}(f(A))} \inf _{\zeta \in f^{-1}(z)}\left\|(\zeta-A)^{-1}\right\|^{-1} .
\end{aligned}
$$

For a simple analytic function $f$ and a small matrix $A$ so that $f(A)$ can be explicitly computed, (2.1) and (2.2) are more convenient for numerical computation. For instance, to calculate $\phi(\epsilon)$, since it is a monotone increasing function of $\epsilon$, we only need to examine points along $\partial \Lambda_{\epsilon}(A)$, the boundary of $\Lambda_{\epsilon}(A)$ :

$$
\sup _{\zeta \in \partial \Lambda_{\epsilon}(A)}\left\|(f(\zeta)-f(A))^{-1}\right\|^{-1} .
$$

(5) The spectral mapping theorem is valid for bounded linear operators on Banach spaces and so is the pseudospectral mapping theorem. For a bounded linear operator $A$ on a Banach space endowed with norm $\|\cdot\|$ and nonnegative number $\epsilon$, the $\epsilon$-pseudospectrum of $A$ is now defined as

$$
\Lambda_{\epsilon}(A) \equiv \overline{\bigcup_{\|E\| \leq \epsilon} \Lambda(A+E)} .
$$

We only demonstrate the first set inclusion of the pseudospectral mapping theorem.

Again, first show that $\phi$ is well defined. Let $\zeta \in \Lambda_{\epsilon}(A)$. Then there exist complex numbers $\left\{\zeta_{n}\right\}$ and bounded linear operators $\left\{E_{n}\right\}$ such that $\zeta_{n} \rightarrow \zeta$ and $\zeta_{n} \in \Lambda\left(A+E_{n}\right)$ with $\left\|E_{n}\right\| \leq \epsilon$. By the spectral mapping theorem,

$$
f\left(\zeta_{n}\right) \in \Lambda\left(f\left(A+E_{n}\right)\right)=\Lambda\left(f(A)+F_{n}\right),
$$

where $F_{n}=f\left(A+E_{n}\right)-f(A)$ and satisfies $\left\|F_{n}\right\| \leq r$ for some positive $r$ independent of $\zeta \in \Lambda_{\epsilon}(A)$. Thus $f(\zeta) \in \Lambda_{r}(f(A))$ which implies that the infimum in the definition of $\phi$ is taken over a nonempty set and so $\phi$ is well defined and $\phi(\epsilon) \leq r$. The first set inclusion now follows directly from the definition of $\phi$.

(6) A result dual to that of the theorem is

$$
\Lambda_{\epsilon}(f(A)) \subset f\left(\Lambda_{\psi(\epsilon)}(A)\right) \subset \Lambda_{\phi(\psi(\epsilon))}(f(A)) .
$$

From this, we obtain immediately that $\epsilon \leq \phi(\psi(\epsilon))$. If in addition $f$ is one-to-one, then apply $f^{-1}$ to the set inclusions in the theorem to get

$$
\Lambda_{\epsilon}(A) \subset f^{-1}\left(\Lambda_{\phi(\epsilon)}(f(A))\right) \subset \Lambda_{\psi(\phi(\epsilon))}(A)
$$

from which $\epsilon \leq \psi(\phi(\epsilon))$ follows. 
(7) In general, it is impossible to have a pseudospectral mapping theorem of the form $f\left(\Lambda_{\epsilon}(A)\right)=\Lambda_{\eta(\epsilon)}(f(A))$ for any $\eta$. To see this, let $A$ be the $1 \times 1$ matrix 1 and $f(z)=z^{2}$. If such a function $\eta$ exists, then

$$
B_{\epsilon}(1)^{2}=B_{\eta(\epsilon)}(1)
$$

where $B_{\epsilon}(z)$ is the closed disk of radius $\epsilon$ with center at $z$ in the complex plane. The right-hand side is a disk of radius $\eta(\epsilon)$ with center at 1 while the left-hand side is clearly not a disk and thus there is a contradiction.

In fact, $f\left(\Lambda_{\epsilon}(A)\right)=\Lambda_{\eta(\epsilon)}(f(A))$ iff $\psi(\phi(\epsilon))=\epsilon$ and $\eta=\phi$.

A natural problem arising from the last remark is to find the class of functions for which the set inclusions in the pseudospectral mapping theorem can be written as an equality for all matrices, as in the case of the spectral mapping theorem. This class turns out to be exactly all the affine functions.

Theorem 2.2. Suppose $f$ is a nonconstant analytic function defined on a nonempty open set $\mathcal{D}$ in the complex plane. Then there exists a nonnegative real-valued function $\eta=\eta(A, \epsilon)$ such that for every matrix $A$ satisfying $\Lambda(A) \subset \mathcal{D}$,

$$
f\left(\Lambda_{\epsilon}(A)\right)=\Lambda_{\eta(A, \epsilon)}(f(A))
$$

for all $\epsilon$ sufficiently small iff $f(z)=\alpha+\beta z$ for some complex numbers $\alpha, \beta$.

Proof. If $f(z)=\alpha+\beta z$, we have already seen that $f\left(\Lambda_{\epsilon}(A)\right)=\Lambda_{\eta(A, \epsilon)}(f(A))$ with $\eta=|\beta| \epsilon$.

Conversely, assume $f$ and $\eta$ are as in the statement of the theorem such that $f\left(\Lambda_{\epsilon}(A)\right)=\Lambda_{\eta(A, \epsilon)}(f(A))$ for all $\epsilon$ sufficiently small and all $A$ such that $\Lambda(A) \subset \mathcal{D}$. Take $A=a$, a constant multiple of the identity matrix with $a \in \mathcal{D}$ and $f^{\prime}(a) \neq 0$. (Recall that $f$ is nonconstant and any zero of $f^{\prime}$ must be isolated.) Then

$$
f\left(B_{\epsilon}(a)\right)=B_{\eta(a, \epsilon)}(f(a)),
$$

where $\epsilon$ is sufficiently small so that $B_{\epsilon}(a) \subset \mathcal{D}$ and $f^{\prime} \neq 0$ on $B_{\epsilon}(a)$. Consequently, $f$ is a continuous one-to-one map from $B_{\epsilon}(a)$ onto $B_{\eta(a, \epsilon)}(f(a))$ with a continuous inverse and so $f$ must map the boundary of the $\epsilon$-disk onto that of the $\eta$-disk. In other words,

$$
f\left(a+\epsilon e^{i \theta}\right)=f(a)+\eta(a, \epsilon) e^{i \omega(a, \epsilon, \theta)}
$$

for all real $\theta$ and some real function $\omega$. Since the left-hand side is a differentiable function of $a, \epsilon$, and $\theta$, so is the right-hand side. Taking the derivative with respect to $\epsilon$, we obtain

$$
e^{i \theta} f^{\prime}\left(a+\epsilon e^{i \theta}\right)=\eta_{\epsilon} e^{i \omega}+\eta e^{i \omega} i \omega_{\epsilon}
$$

or

$$
f^{\prime}\left(a+\epsilon e^{i \theta}\right)=e^{i(\omega-\theta)}\left(\eta_{\epsilon}+i \eta \omega_{\epsilon}\right)
$$

Now take the derivative of (2.5) with respect to $\theta$ to get

$$
f^{\prime}\left(a+\epsilon e^{i \theta}\right)=e^{i(\omega-\theta)} \frac{\eta \omega_{\theta}}{\epsilon} .
$$

Comparing these two equations, we deduce that

$$
\eta_{\epsilon}+i \eta \omega_{\epsilon}=\frac{\eta \omega_{\theta}}{\epsilon} .
$$


Noting that both $\eta$ and $\omega$ are real, this is possible only if $\omega$ is independent of $\epsilon$. Hence

$$
\omega_{\theta}=\frac{\eta_{\epsilon} \epsilon}{\eta} .
$$

Since the left-hand side is independent of $\epsilon$ while the right-hand side is independent of $\theta$, the above must be equal to some real $p$ which is independent of both $\theta$ and $\epsilon$. Thus $\omega=p \theta+q$ for some real $q$ to be determined. Because $e^{i \omega}$ is $2 \pi$-periodic in $\theta, p$ must be an integer. In this case, it cannot depend on $a$ since $\omega$ is continuous. Thus we write

$$
\omega=p \theta+q(a) .
$$

Now solve for $\eta$ in (2.6) to obtain

$$
\eta=c(a) \epsilon^{p},
$$

where $c(a)$ is positive. The continuity of $\eta$ at $\epsilon=0$ implies that $p \geq 1$.

Now take the derivative of (2.5) with respect to $a$ to obtain

$$
\left(f^{\prime}\left(a+\epsilon e^{i \theta}\right)-f^{\prime}(a)\right) e^{-i(p \theta+q(a))}=\epsilon^{p}\left(c_{a}+i c q_{a}\right)
$$

after some simplifications. For all $\epsilon$ sufficiently small, this is equivalent to

$$
\sum_{k=1}^{\infty} \frac{f^{(k+1)}(a) e^{-i q(a)}}{k !} \epsilon^{k} e^{i \theta(k-p)}=\epsilon^{p}\left(c_{a}+i c q_{a}\right) .
$$

Since the right-hand side is independent of $\theta$, we may conclude that when $k \neq p$ and $k \geq 1, f^{(k+1)}(a)=0$, while if $k=p$,

$$
\frac{f^{(k+1)}(a) e^{-i q(a)}}{k !}=c_{a}+i c q_{a} .
$$

In summary, we have deduced that $f(a)=\alpha+\beta a+\mu a^{p+1}$ for some complex constants $\alpha, \beta, \mu$. From (2.4),

$$
\alpha+\beta B_{\epsilon}(a)+\mu B_{\epsilon}(a)^{p+1}=B_{\eta(a, \epsilon)}\left(\alpha+\beta a+\mu a^{p+1}\right) .
$$

For any nonzero $a$, if $\mu \neq 0$, then the left-hand side is not a disk while the righthand side is and we have a contradiction. Consequently, $f(z)=\alpha+\beta z$ and the proof is completed.

\section{WEAK PSEUdOSPECTRAL MAPPING THEOREM}

The functions $\phi$ and $\psi$ are continuous and monotone increasing but it appears to be difficult to deduce other properties. We can derive simpler functions which give an upper bound to $\phi$ and to $\psi$, leading to a weak pseudospectral mapping theorem. One consequence of this is a simple approximate upper bound to $\psi(\phi(\epsilon))$ in terms of a condition number for small $\epsilon$.

Theorem 3.1. Let $A$ be a matrix and $f$ be an analytic function defined on an open set containing $\Lambda(A)$. For each $\epsilon \geq 0$ and sufficiently small, define

$$
\gamma(\epsilon)=\sup _{\|E\| \leq \epsilon}\|f(A+E)-f(A)\| .
$$

Then

$$
f\left(\Lambda_{\epsilon}(A)\right) \subset \Lambda_{\gamma(\epsilon)}(f(A)) \subset f\left(\Lambda_{\delta(\gamma(\epsilon))}(A)\right),
$$

where $\delta$ is defined in (3.2) and (3.3) below. 
Proof. We first show that $f\left(\Lambda_{\epsilon}(A)\right) \subset \Lambda_{\gamma(\epsilon)}(f(A))$. Let $z \in f\left(\Lambda_{\epsilon}(A)\right)$. Then there is some complex number $\zeta$ and matrix $E$ such that $z=f(\zeta),\|E\| \leq \epsilon$, and $\zeta \in \Lambda(A+E)$. By the spectral mapping theorem,

$$
f(\zeta) \in \Lambda(f(A+E))=\Lambda(f(A)+F),
$$

where $F=f(A+E)-f(A)$ and satisfies $\|F\| \leq \gamma(\epsilon)$. Thus $z \in \Lambda_{\gamma(\epsilon)}(f(A))$.

Now we prove the second set inclusion. First, assume that $D f(A)$ is nonsingular. Here, $D f(A)$ is the derivative of $f$ evaluated at $A$ and is a linear operator from the space of matrices onto itself. Define $\mathcal{G}(B, C)=f(A+B)-f(A)-C$ for matrices $B$ and $C$. Note $\mathcal{G}(0,0)=0, D_{B} \mathcal{G}(0,0)=D f(A)$ which is nonsingular. By the implicit function theorem, there is an analytic matrix function $\mathcal{E}$ and a positive constant $\alpha$ such that $\mathcal{G}(\mathcal{E}(C), C)=0$ for all $\|C\| \leq \alpha$ and $\mathcal{E}(0)=0$. Define

$$
\delta(s)=\sup _{\|C\| \leq s}\|\mathcal{E}(C)\|, \quad s \leq \alpha .
$$

Suppose $z \in \Lambda_{\gamma(\epsilon)}(f(A))$. Let $F$ be a matrix such that $\|F\| \leq \gamma(\epsilon)$ and $z \in$ $\Lambda(f(A)+F)$. It is assumed that $\epsilon$ is small enough such that $\gamma(\epsilon) \leq \alpha$. Let $E=\mathcal{E}(F)$. Note $f(A+E)-f(A)=F$ and $\|E\| \leq \delta(\gamma(\epsilon))$. Now $z \in \Lambda(f(A+E))$ and by the spectral mapping theorem, $z=f(\zeta)$ for some complex number $\zeta \in \Lambda(A+E)$ or $\zeta \in \Lambda_{\delta(\gamma(\epsilon))}(A)$. This implies that $z \in f\left(\Lambda_{\delta(\gamma(\epsilon))}(A)\right)$.

To complete the proof, we consider the case when $\operatorname{Df}(A)$ is singular. Define $g(z)=f(z)+\beta z$, where $\beta$ is any small positive constant such that $D g(A)=$ $D f(A)+\beta$ is nonsingular. By the result of the above paragraph,

$$
\Lambda_{\tilde{\gamma}(\epsilon)}(g(A)) \subset g\left(\Lambda_{\tilde{\delta}(\tilde{\gamma}(\epsilon))}(A)\right),
$$

where $\tilde{\gamma}$ and $\tilde{\delta}$ are defined as in (3.1) and (3.2) with $f$ replaced by $g$. Thus

$$
\Lambda_{\tilde{\gamma}(\epsilon)}(f(A)+\beta A) \subset f\left(\Lambda_{\tilde{\delta}(\tilde{\gamma}(\epsilon))}(A)\right)+\beta \Lambda_{\tilde{\delta}(\tilde{\gamma}(\epsilon))}(A) .
$$

Note $\lim _{\beta \rightarrow 0} \tilde{\gamma}(\epsilon)=\gamma(\epsilon)$. By an implicit function argument, for a fixed $s$,

$$
\delta(s) \equiv \lim _{\beta \rightarrow 0} \tilde{\delta}(s)
$$

is well defined. Consequently,

$$
\Lambda_{\gamma(\epsilon)}(f(A)) \subset f\left(\Lambda_{\delta(\gamma(\epsilon))}(A)\right)
$$

and the proof is complete.

Here are some more remarks.

(1) Note that $\gamma(0)=0=\delta(0)$ and thus the theorem also reduces to the usual spectral mapping theorem when $\epsilon=0$.

(2) If $f(z)=\alpha+\beta z$, where $\alpha, \beta$ are complex numbers, then $\gamma(\epsilon)=|\beta| \epsilon$. Also, $\mathcal{G}(B, C)=0$ is equivalent to $\beta B-C=0$. Consequently, $\delta(s)=s /|\beta|$ and

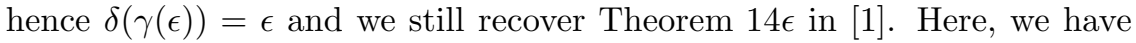
assumed $\beta \neq 0$; otherwise, it is trivially true.

(3) Does the weak pseudospectral mapping theorem generalize to the case if $A$ is a bounded linear operator on a Banach space? It is not difficult to check that the first set inclusion still holds. The second set inclusion holds provided that $D f(A)$ is assumed to be one-to-one and onto. Then its inverse is bounded so that the implicit function theorem can still be invoked and the previous proof carries over. When this assumption is not valid, our 
method of perturbation by $\beta$ may not work. This is because it is possible that some open set containing zero is a subset of $\Lambda(D f(A))$. It is not clear how to proceed in this case.

(4) For simple functions such as $f(z)=z^{2}$ or $e^{z}$, we can obtain an explicit expression for $\mathcal{E}(C)$, facilitating the calculation of $\delta$. (See the example below and Section 5.) However, this is not possible in general.

(5) A simple approximation of an upper bound of $\gamma(\epsilon)$ when $\epsilon$ is small is $\|D f(A)\| \epsilon$. From the equation $f(A+\mathcal{E}(C))-f(A)=C$, we obtain $D f(A) \mathcal{E}(C) \approx C$ or $\|\mathcal{E}(C)\| \leq\left\|D f(A)^{-1}\right\|\|C\|$ approximately. Thus an approximate upper bound of $\delta(\gamma(\epsilon))$ is $\kappa(D f(A)) \epsilon$, where $\kappa()$ is the condition number of a matrix. Consequently,

$$
f\left(\Lambda_{\epsilon}(A)\right) \subset \Lambda_{\|D f(A)\| \epsilon}(f(A)) \subset f\left(\Lambda_{\kappa(D f(A)) \epsilon}(A)\right)
$$

holds approximately when $D f(A)$ is nonsingular. Since $\phi \leq \gamma$ and $\psi \leq \delta$, we have

$$
\psi(\phi(\epsilon)) \leq \kappa(D f(A)) \epsilon
$$

approximately or $\psi(\phi(\epsilon)) \leq \kappa(D f(A)) \epsilon+O\left(\epsilon^{2}\right)$.

Alternatively, the following is another simple estimate:

$$
f\left(\Lambda_{\epsilon}(A)\right) \subset \Lambda_{c_{1} \epsilon}(f(A)) \subset f\left(\Lambda_{c_{1} c_{2} \epsilon}(A)\right),
$$

where

$$
c_{1}=c_{1}(\epsilon)=\sup _{\|E\| \leq \epsilon}\|D f(A+E)\|, \quad c_{2}=c_{2}(\epsilon)=\sup _{\|E\| \leq \epsilon}\left\|D f(A+E)^{-1}\right\| .
$$

We now give an example. Let $A$ be the $1 \times 1$ matrix 1 and $f(z)=z^{2}$. Then

$$
\gamma(\epsilon)=\sup _{|b| \leq \epsilon}\left|(1+b)^{2}-1^{2}\right|=2 \epsilon+\epsilon^{2}
$$

Note $\mathcal{G}(b, c)=(1+b)^{2}-1^{2}-c=0$ implies that $b=\mathcal{E}(c)=-1 \pm \sqrt{1+c}$. Since $\mathcal{E}(0)=0$, we discard the "-" branch of solutions. As a result,

$$
\delta(s)=\sup _{|c| \leq s}|-1+\sqrt{1+c}|=1-\sqrt{1-s}
$$

and so $\delta(\gamma(\epsilon))=1-\sqrt{1-2 \epsilon-\epsilon^{2}}$. See Figure 3.1 for the boundaries of the sets $B_{\epsilon}(1)^{2}, B_{2 \epsilon+\epsilon^{2}}(1), B_{1-\sqrt{1-2 \epsilon-\epsilon^{2}}}(1)^{2}$ when $\epsilon=0.4$. As we shall see, the result of the theorem is sharp for one-dimensional matrices, that is, $\phi=\gamma$ and $\psi=\delta$.

If we take $A=0$ instead, then since $f^{\prime}(0)=0$, the calculation of $\delta$ involves an extra step. The equation $\mathcal{G}(b, c)=0$ becomes $b^{2}+\beta b-c=0$ which implies that

$$
b=\mathcal{E}(c)=\frac{-\beta \pm \sqrt{\beta^{2}+4 c}}{2} .
$$

Note that both branches are valid this time because the requirement is that $\mathcal{E}(0)=0$ at $\beta=0$. Consequently,

$$
\begin{aligned}
\delta(s) & =\lim _{\beta \rightarrow 0} \sup _{|c| \leq s}|\mathcal{E}(c)| \\
& =\frac{1}{2} \lim _{\beta \rightarrow 0}\left|\beta \mp \sqrt{\beta^{2} \mp 4 s}\right| \\
& =\sqrt{s} .
\end{aligned}
$$

With $\gamma(\epsilon)=\epsilon^{2}$, we obtain the ideal result $\delta(\gamma(\epsilon))=\epsilon$. For such examples, it is simpler to compute $\gamma$ and $\delta$ than $\phi$ and $\psi$ even though they give the same answer. 


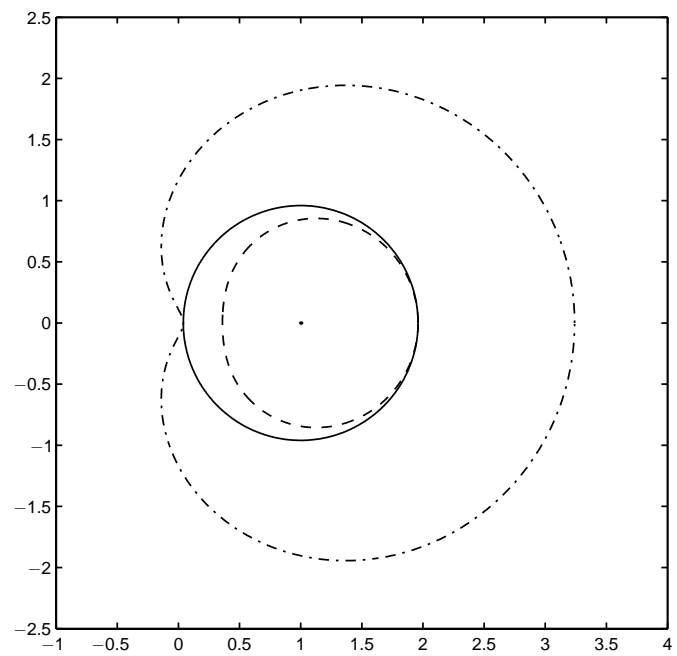

FiguRE 3.1. Relationship among the three sets in the (general or weak) pseudospectral mapping theorem for a $1 \times 1$ matrix.

In conclusion, the weak pseudospectral mapping theorem gives the bounds $\phi(\epsilon) \leq$ $\|D f(A)\| \epsilon+O\left(\epsilon^{2}\right)$ and $\psi(\phi(\epsilon)) \leq \kappa(D f(A)) \epsilon+O\left(\epsilon^{2}\right)$.

\section{Pseudospectral mapping theorem for normal matrices}

When $A$ is normal with eigenvalues $\left\{\lambda_{j}\right\}$, the result of the weak pseudospectral mapping theorem simplifies to

$$
\bigcup_{j} f\left(B_{\epsilon}\left(\lambda_{j}\right)\right) \subset \bigcup_{j} B_{\gamma(\epsilon)}\left(f\left(\lambda_{j}\right)\right) \subset \bigcup_{j} f\left(B_{\delta(\gamma(\epsilon))}\left(\lambda_{j}\right)\right) .
$$

Unfortunately, it does not appear to be possible to obtain an explicit expression for $\gamma$ and $\delta$ and so the above relations cannot be simplified further. The situation does not improve even when $A=0$, except in the one-dimensional case. This motivates us to formulate a pseudospectral mapping theorem for normal matrices.

Theorem 4.1. Let $A$ be a normal matrix and $f$ be an analytic function defined on an open set containing $\Lambda(A)=\left\{\lambda_{j}\right\}$. For each $\epsilon \geq 0$ and sufficiently small,

$$
\bigcup_{j} f\left(B_{\epsilon}\left(\lambda_{j}\right)\right) \subset \bigcup_{j} B_{\gamma_{j}(\epsilon)}\left(f\left(\lambda_{j}\right)\right) \subset \bigcup_{j} f\left(B_{\delta_{j}\left(\gamma_{j}(\epsilon)\right)}\left(\lambda_{j}\right)\right),
$$

where

$$
\gamma_{j}(\epsilon)=\sup _{|c| \leq \epsilon}\left|f\left(\lambda_{j}+c\right)-f\left(\lambda_{j}\right)\right|
$$

and $\delta_{j}$ is defined in (4.1) and (4.2) below. The functions $\gamma_{j}$ and $\delta_{j}$ cannot be replaced by smaller ones.

Proof. The proof of the set inclusions is exactly the same as before except that it is simpler because we only need to work on scalar quantities. If $f^{\prime}\left(\lambda_{j}\right) \neq 0$, then the 

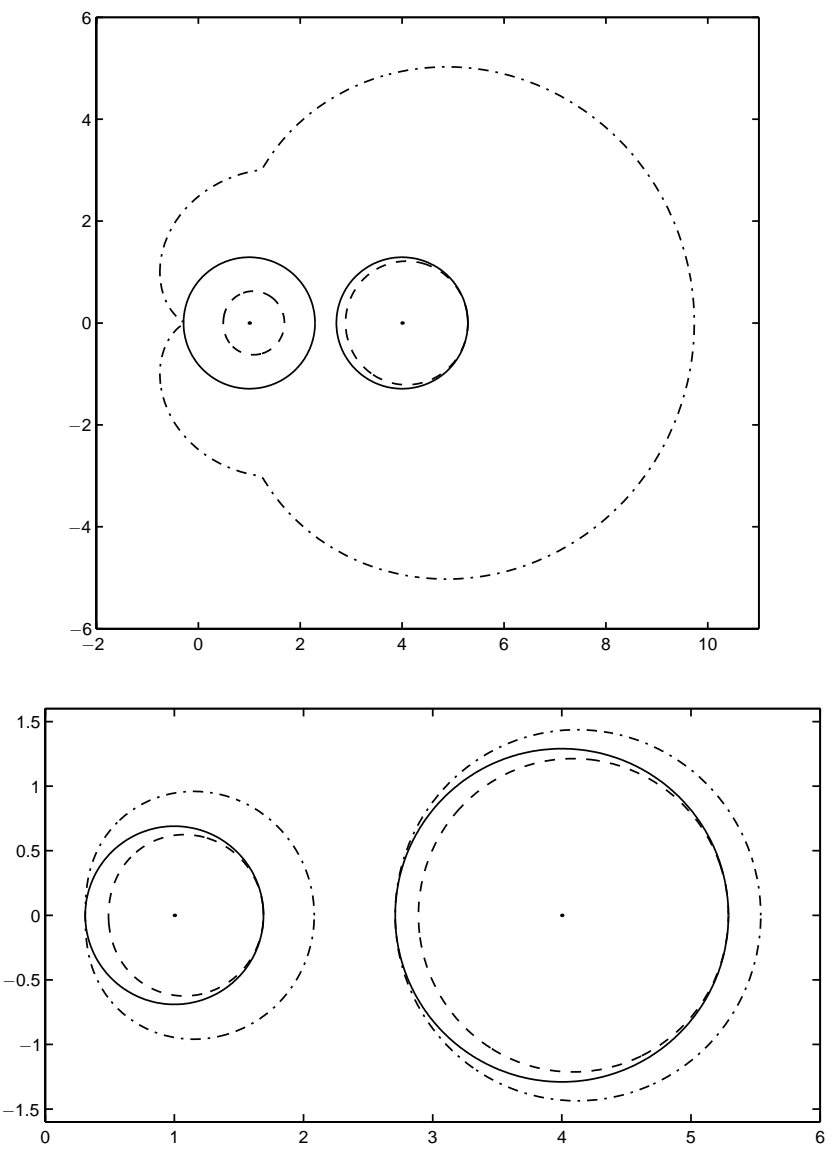

Figure 4.1. Three relevant sets in the general pseudospectral mapping theorem for a $2 \times 2$ diagonal matrix (top) and corresponding sets from the pseudospectral mapping theorem for normal matrices (bottom).

equation $f\left(\lambda_{j}+b\right)-f\left(\lambda_{j}\right)-c=0$ has a unique solution $b=\mathcal{E}_{j}(c)$ for all numbers $c$ in some neighborhood of 0 . Define

$$
\delta_{j}(s)=\sup _{|c| \leq s}\left|\mathcal{E}_{j}(c)\right| .
$$

In case $f^{\prime}\left(\lambda_{j}\right)=0$, as before, we perturb $f(z)$ by $\beta z$, giving rise to a function $\tilde{\delta}_{j}$ corresponding to (4.1). Now define

$$
\delta_{j}(s)=\lim _{\beta \rightarrow 0} \tilde{\delta}_{j}(s)
$$

and proceed as before.

To show that the set inclusions are sharp, it is sufficient to consider $1 \times 1$ matrices. For the first set inclusion, suppose there exist numbers $a, \epsilon>0$ and small, $r<$ $\gamma(\epsilon)$ such that $f\left(B_{\epsilon}(a)\right) \subset B_{r}(f(a))$. Let $c$ be a number such that $|c| \leq \epsilon$ and $|f(a+c)-f(a)|=\gamma(\epsilon)>r$. This implies that $f(a+c) \in B_{\gamma(\epsilon)}(f(a)) \backslash B_{r}(f(a))$. Since $a+c \in B_{\epsilon}(a), f(a+c) \in f\left(B_{\epsilon}(a)\right) \subset B_{r}(f(a))$, which is a contradiction. 
For the second set inclusion, suppose there is some $r<\delta(\gamma(\epsilon))$ such that $B_{\gamma(\epsilon)}(f(a)) \subset f\left(B_{r}(a)\right)$. Assume $f^{\prime}(a) \neq 0$. Let $c$ be any number such that $|c| \leq \gamma(\epsilon)$ and $b=\mathcal{E}(c)$ satisfies $|b|=\delta(\gamma(\epsilon))$. Thus $f(a+b)=f(a)+c \epsilon$ $B_{\gamma(\epsilon)}(f(a)) \subset f\left(B_{r}(a)\right)$ or $|b| \leq r<\delta(\gamma(\epsilon))$. (Here, we assume that $\epsilon$ is small such that $f^{\prime} \neq 0$ on $B_{\delta(\gamma(\epsilon))}(a)$ and so the inverse of $f$ exists on $f\left(B_{\delta(\gamma(\epsilon))}(a)\right)$.) This contradicts the definition of $b$. The case $f^{\prime}(a)=0$ follows from the above and taking of limits. This completes the proof.

Hence when $A$ is an $n \times n$ normal matrix, the problem decouples into $n$ (or fewer if there are multiple eigenvalues) scalar problems of estimating $\gamma_{j}$ and $\delta_{j}$. It is now clear that the set inclusions in the general pseudospectral mapping theorem can be unsatisfactory. For normal matrices, one requires functions $\gamma_{j}$ and $\delta_{j}$ to accurately describe the region around $\lambda_{j}$ while in the general theorem, only one $\phi$ and one $\psi$ are available for the whole spectrum. See Figure 4.1 for an example of a $2 \times 2$ diagonal matrix $A$ with diagonal entries 1 and 2. We take $f(z)=z^{2}$ and $\epsilon=0.3$. It is obvious that $\Lambda_{\psi(\phi(0.3))}(A)^{2}$ is quite large compared to the corresponding sets in the pseudospectral mapping theorem for normal matrices.

This section can be summarized by the statement that $\phi_{j}=\gamma_{j}$ and $\psi_{j}=\delta_{j}$ for normal matrices where $\phi_{j}$ and $\psi_{j}$ are defined in the obvious way.

\section{NumericAl RESUltS}

We report on some MATLAB numerical experiments for the $10 \times 10$ Grcar matrix $A_{G}$ and Kahan matrix $A_{K}$ defined as

$$
\begin{aligned}
A_{G} & =\left[\begin{array}{ccccccc}
1 & 1 & 1 & 1 & & & \\
-1 & 1 & 1 & 1 & 1 & & \\
& \ddots & \ddots & \ddots & \ddots & \ddots & \\
& & -1 & 1 & 1 & 1 & 1 \\
& & & -1 & 1 & 1 & 1 \\
& & & & -1 & 1 & 1 \\
& & & & & -1 & 1
\end{array}\right], \\
A_{K} & =\left[\begin{array}{cccccc}
1 & -c & \ldots & \ldots & \cdots & -c \\
& s & -s c & \cdots & \cdots & -s c \\
& & s^{2} & -s^{2} c & \cdots & -s^{2} c \\
& & & \ddots & & \vdots \\
& & & & s^{8} & -s^{8} c \\
& & & & & s^{9}
\end{array}\right],
\end{aligned}
$$

where $s^{9}=0.1$ and $c=\sqrt{1-s^{2}}$. First consider $A=A_{G}$ with $f(z)=z^{2}$ and $\epsilon=0.01$. As mentioned before, we compute $\phi(\epsilon)$ using (2.3), where the supremum is taken for $\zeta$ along the boundary of $\Lambda_{\epsilon}(A)$. Similarly, $\psi$ is computed via (2.2) with the supremum taken over the boundary of $\Lambda_{s}(f(A))$. For $\gamma$ and $\delta$, we take a sample of 100, 000 random matrices whose entries are independent and come from the normal distribution of mean zero and variance one. Each matrix is rescaled to have norm $\epsilon$ and $\gamma$ is estimated by the maximum of $\|f(A+E)-f(A)\|$ with $E$ ranging over the sample of random matrices. Similarly, for each $E$ now rescaled to have norm $\gamma$, compute $\mathcal{E}(E)=-A+\sqrt{A^{2}+E}$ and take $\delta$ as the maximum of $\{\|\mathcal{E}(E)\|\}$. We find that $(\phi(\epsilon), \psi(\phi(\epsilon)), \gamma(\epsilon), \delta(\gamma(\epsilon)))=(0.043,0.014,0.051,0.43)$. 

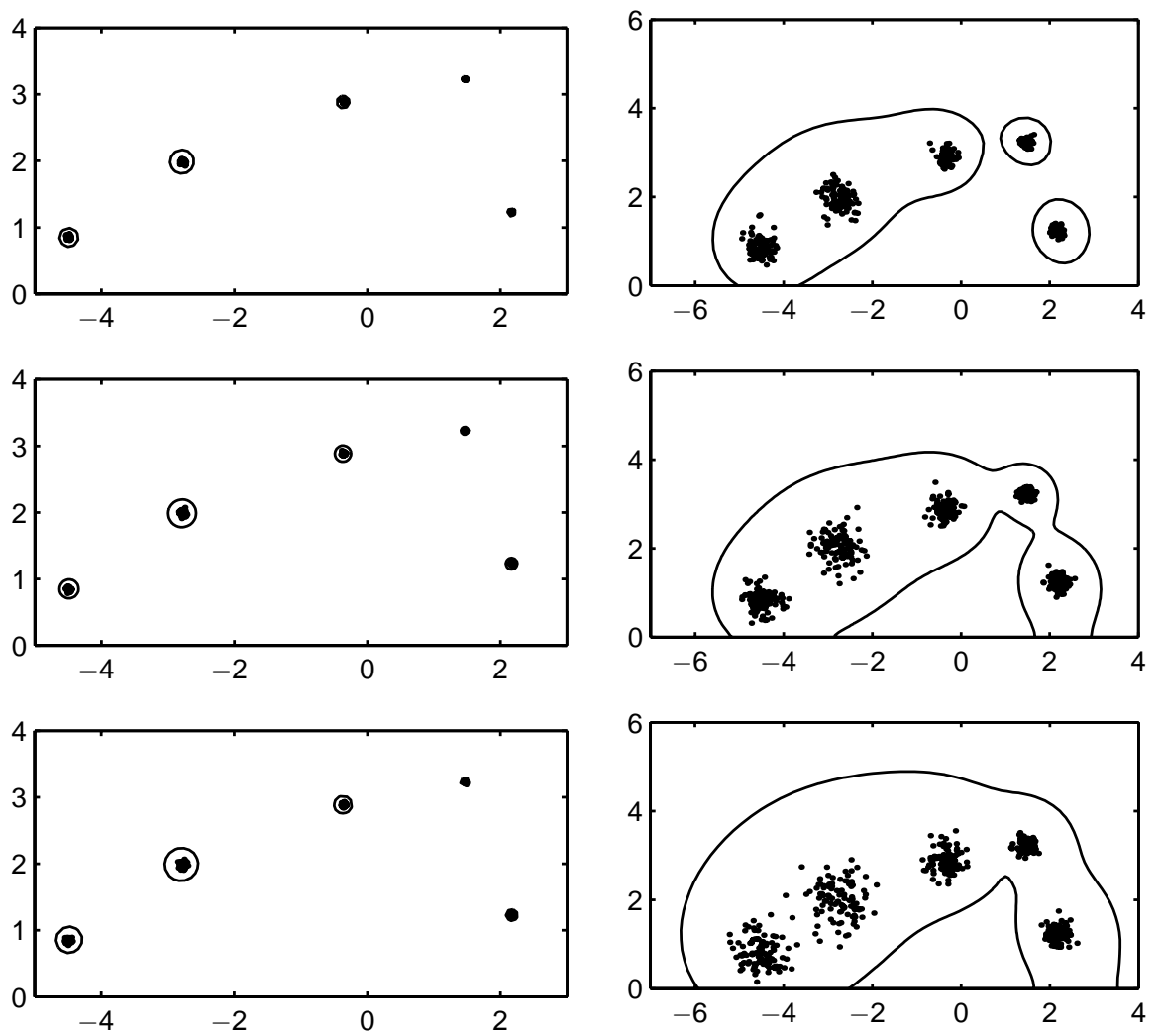

(a) $\epsilon=0.01$

(b) $\epsilon=0.1$

Figure 5.1. Relationship among the three sets in the general pseudospectral mapping theorem for the Grcar matrix $A$ : $\Lambda_{\epsilon}(A)^{2} \subset \Lambda_{\phi(\epsilon)}\left(A^{2}\right) \subset \Lambda_{\psi(\phi(\epsilon))}(A)^{2}$ from top to bottom.

With $\epsilon=0.1$, the parameter vector becomes $(0.48,0.18,0.51,166)$. This example shows that $\delta$ can be quite a poor approximation to $\psi$ when $\epsilon$ is large. Roughly speaking, this is due to the fact that $\left\|D f^{-1}\right\|$ is large. The relevant sets in the upper half-plane are plotted in Figure [5.1] If $f(z)=e^{z}$, then $\mathcal{E}(E)=\ln \left(e^{A}+E\right)-A$. Everything is qualitatively similar.

We now give some details regarding the rather straightforward computations to obtain the figures. We compute and display $f\left(\partial \Lambda_{\epsilon}(A)\right)$ instead of $f\left(\Lambda_{\epsilon}(A)\right)$. Toward this end, the excellent code psa.m ([5]) is used to compute $\partial \Lambda_{\epsilon}(A)$ and then $f\left(\partial \Lambda_{\epsilon}(A)\right)$ is calculated directly. With respect to $\partial \Lambda_{\phi(\epsilon)}(f(A))$, we first compute $f(A)$ and $\phi(\epsilon)$ and then use psa.m to calculate $\partial \Lambda_{\phi(\epsilon)}(f(A))$. We display as well $\{f(\lambda)\}$, where $\{\lambda\}$ is the set of eigenvalues of $A+E$ generated from 100 random matrices $E$. These appear as dots in the top diagrams of the figure. This method is clearly inferior to the procedure described above but offers a check of validity of our method. Similarly, we compute the dots in the middle and bottom diagrams by this perturbation method. 

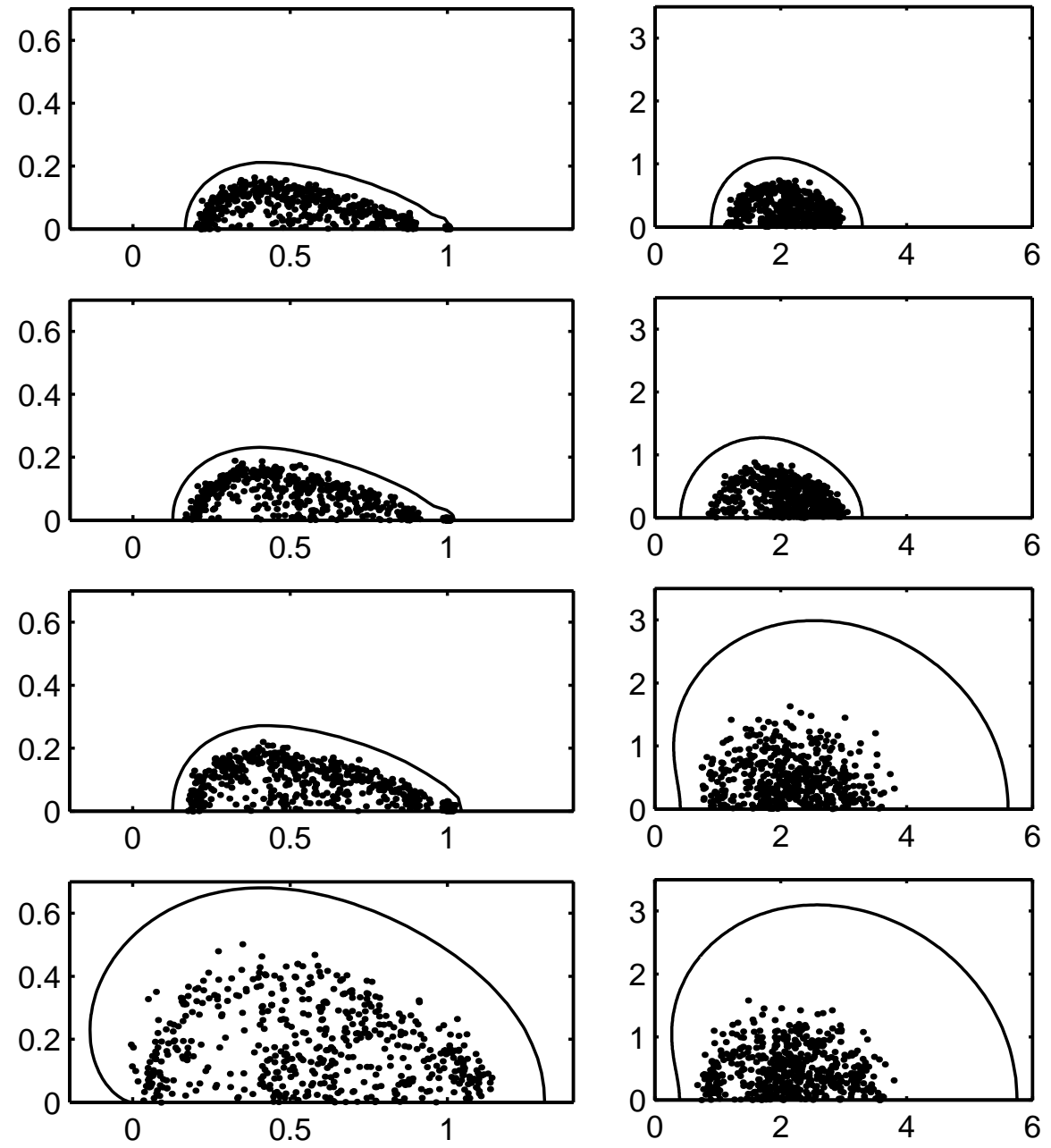

(a) $f(z)=z^{2}, \epsilon=0.001$

(b) $f(z)=e^{z}, \epsilon=0.1$

FiguRE 5.2. Relationship among the four sets in the pseudospectral mapping theorems for the Kahan matrix $A: f\left(\Lambda_{\epsilon}(A)\right) \subset$ $\Lambda_{\phi(\epsilon)}(f(A)) \subset f\left(\Lambda_{\psi(\phi(\epsilon))}(A)\right) \subset f\left(\Lambda_{\delta(\gamma(\epsilon))}(A)\right)$ from top to bottom.

The plots for the Kahan matrix are given in Figure 5.2. When $f(z)=z^{2}, \epsilon=$ 0.001 , the computed value of the parameter vector is $(0.0019,0.0027,0.0027,0.062)$ while it is $(0.32,0.60,0.32,0.63)$ for $f(z)=e^{z}, \epsilon=0.1$. In the last example, $\delta$ is a good approximation to $\psi$. For most of these experiments, $\psi(\phi(\epsilon))$ is not much bigger than $\epsilon$ and this makes the general pseudospectral mapping theorem rather effective. 


\section{ACKNOWLEDGMENTS}

It is my pleasure to thank Nick Trefethen for several very beneficial discussions and for his interest in this work. I am also grateful to Mark Embree and Thomas Wright for their invaluable help in generating the MATLAB plots in this paper and to Mark for discussions and comments on an earlier draft of this paper.

\section{REFERENCES}

[1] M. Embree and L. N. Trefethen. Generalizing eigenvalue theorems to pseudospectra theorems. SIAM J. Sci. Comput., 23:583-590, 2001. MR 2002k:15019

[2] L. N. Trefethen. Pseudospectra of linear operators. SIAM Rev., 39:383-406, 1997. MR 98i:47004

[3] L. N. Trefethen. Spectra and pseudospectra: The behavior of nonnormal matrices and operators. In M. Ainsworth, J. Levesley, and M. Marletta, editors, The Graduate Student's Guide to Numerical Analysis, pages 217-250. Springer-Verlag, Berlin, 1998.

[4] L. N. Trefethen. Computation of pseudospectra. Acta Numer., 8:247-295, 1999. MR 2002b:65062

[5] T. G. Wright and L. N. Trefethen. Large-scale computation of pseudospectra using ARPACK and Eigs. SIAM J. Sci. Comput., 23:591-605, 2001. MR 2002h:65061

Department of Mathematics, University of Manitoba, Winnipeg, Manitoba, Canada R3T $2 \mathrm{~N} 2$

E-mail address: luish@cc.umanitoba.ca 